\title{
HORMONES CAN INFLUENCE ANTIBIOTIC SUSCEPTIBILITIES EVEN IN MONO- AND CO-CULTURE CONDITIONS
}

\author{
Fatma Kalayc1-YÜKSEK ${ }^{1 *}$, Defne GÜMÜŞ, Mine ANĞ-KÜÇÜKER \\ ${ }^{1}$ Istanbul Yeni Yüzyıl University, Faculty of Medicine, Department of Medical Microbiology, Istanbul, \\ Turkey
}

*Correspondence:

Fatma KALAYCI-YÜKSEK

fatma.kalayci@yeniyuzyil.edu.tr

Received: 12 November 2021; Accepted: 10 December 2021; Published: 30 December 2021

\begin{abstract}
Pseudomonas aeruginosa and Staphylococcus aureus are known as important nosocomial infectious agents also their co-infections are commonly seen in some patient groups. It is well known that host factors such as hormones have roles in modulation of growth, pathogenesis and susceptibilities to antimicrobials. In our study, the influences of norepinephrine (NE) and melatonin (MEL) on antibiotic susceptibilities were examined in mono and co-culture conditions. Methicilin resistant Staphylococcus aureus (MRSA) ATCC 43300 and Pseudomonas aeruginosa ATCC 27853 were investigated to determine the minimum inhibitory concentrations (MIC) and minimum bactericidal concentrations (MBC) of ciprofloxacin and gentamicin in the absence/presence of NE $(0.0017$ and $0.04 \mu \mathrm{g} / \mathrm{mL})$ and MEL (6 and 60 $\mathrm{pg} / \mathrm{mL}$ ) by microdilution method in mono and co-culture. It was found that hormones decreased (among 2-64 fold) MIC and MBC values of both antibiotics for MRSA. However, it was shown that hormones had no effect on MIC values of both antibiotics for P. aeruginosa. MIC and MBC values of both antibiotics for coculture were found to be reduced compared to monoculture of MRSA; were found to be increased compared to monoculture of $P$. aeruginosa. Whereas, hormones decreased MIC values of both antibiotics in co-culture conditions. Our results suggest that both hormones decreased MIC values and it seems that hormones could influence antibiotic susceptibilities in a strain-dependent manner.
\end{abstract}

Keywords: Pseudomonas aeruginosa and Staphylococcus aureus mono and co-culture, norepinephrine, melatonin, MIC, MBC, gentamicin, ciprofloxacin, antibiotic susceptibility

\section{Introduction}

It is well known that methicilin resistant Staphylococcus aureus (MRSA) and Pseudomonas aeruginosa are the most common hospital-acquired infectious agents; and their co-infections are very prevalent and harmful in some clinical outcomes such as cystic fibrosis, chronic wound infections, ulcers, surgical site infections (Dalton et al., 2011; DeLeon et al., 2014; Murray et al., 2014; Nguyen and Oglesby-Sherrouse, 2016; Briaud et al., 2020b). In recent years, there are many studies focusing on alterations of biological properties of $P$. aeruginosa and S. aureus when they are co-existed (Yang et al., 2011; Kim et al., 2015; Ping et al., 2017; Alves et al., 2018; Kahl, 2018; Wijesinghe et al., 2019; Briaud et al., 2020b; Yung et al., 2021); also for treatment of these complex infections, development of some alternative treatment 
strategies are gained importance (Kahl, 2018; Yung et al., 2021).

On the other hand, many studies have shown the effects of host hormones, as environmental factors in the host body, on bacterial physiology during the infectious processes (Plotkin and Viselli, 2000; Plotkin et al., 2003; Lyte Mark, 2010; Lyte, Mark, Cryan, 2014; Gumus et al., 2017; Gümüş et al., 2019; Gonçalves et al., 2020; Boukerb et al., 2021; Engelsöy et al., 2021). These studies highlighted a new concept which is known as microbial endocrinology, a bi-directional interaction between host and microbe through interkingdom signaling based on neuroendocrine factors of human and sensor molecules of microorganisms (Lyte Mark, 2010; Lyte, Mark, Cryan, 2014). According to previous studies, microorganisms sense and respond to hormones and regulate their selfgrowth, virulence, antimicrobial susceptibilities (Freestone et al., 2007; Fteita et al., 2014; Sandrini et al., 2014; Yang et al., 2014; Gumus et al., 2017; Gümüş et al., 2019; Truccollo et al., 2020; Engelsöy et al., 2021; Lyte et al., 2021).

From past to present, most of these studies focused on the interactions between norepinephrine and microorganisms (Belay et al., 2003; Bansal et al., 2007; Freestone et al., 2007; Lyte Mark, 2010; Yang et al., 2014; Lyte, Mark, Cryan, 2014; Sandrini et al., 2014; $\mathrm{Xu}$ et al., 2015; Bearson, 2016; Boyanova, 2017; Gümüş et al., 2019; Truccollo et al., 2020; Lucca et al., 2020). It has been reported that norepinephrine affects the behaviors of microorganisms via regulating iron accessing and/or triggers of norepinephrine-induced autoinducers production (Freestone et al., 2007; Li W, Lyte M, Freestone PP, Ajmal A, ColmerHamood J, 2009; Lyte Mark, 2010; Lyte, Mark, Cryan, 2014; Sandrini et al., 2014).

Another mammalian hormone melatonin, have a capacity of binding some metal molecules, such as iron, zinc and copper; it was hypothesized that in this way, melatonin influences microorganism' cytoplasmic regulation (Zhou et al., 2016; Liu et al., 2020; He et al., 2021).

In the present study, we aimed to investigate the roles of norepinephrine (NE) and melatonin (MEL) on antimicrobial susceptibilities of methicillin-resistant Staphylococcus aureus and Pseudomonas aeruginosa in single and co-culture conditions.

\section{Materials and Methods}

\subsection{Bacteria}

We investigated the susceptibilities of MRSA ATCC 43300 and $P$. aeruginosa ATCC 27853 to ciprofloxacin (CIP) and gentamicin (GN) in the presence/absence of NE and MEL using the microdilution method.

\subsection{Hormones}

We examined two concentrations of norepinephrine (LNE: 0.0017 and HNE: 0.04 $\mu \mathrm{g} / \mathrm{mL}$ )] and melatonin (LMEL: 6 and HMEL: $60 \mathrm{pg} / \mathrm{mL}$ ) according to their physiological blood levels.

\subsection{Antibiotics, Determination of Minimum Inhibition Concentrations (MIC)}

MRSA and $P$. aeruginosa were grown in Mueller Hinton broth at $37{ }^{\circ} \mathrm{C}$ for 24 hours to provide overnight culture. The initial concentrations of bacteria were arranged to approximately $10^{7} \quad \mathrm{CFU} / \mathrm{mL}$. Minimum inhibition concentrations were determined by microdilution method. To obtain a co-culture condition, MRSA and $P$. aeruginosa were added into the same well simultaneously.

Two-fold serial concentrations were prepared between 2.5- $1280 \mu \mathrm{g} / \mathrm{mL}$ for ciprofloxacin and gentamicin in 96-well plates. (CLSI, 2021) CIP and GN were selected 
because they are broad-spectrum antibiotics and used widely.

Hormones $(20 \mu \mathrm{L})$ and bacteria (as cocultured or separately) $(20 \mu \mathrm{L})$ were added to wells. The plates were incubated at $37{ }^{\circ} \mathrm{C}$ for 24 hours to examine whether the presence of hormones could influence the MIC values. MIC values were considered as the lowest concentration of the antimicrobial agent which inhibited the growth of the microorganism. The experiments were repeated three times independently and all conditions were analyzed twice.

\subsection{The Determination of Minimum Bactericidal Concentrations (MBC)}

After determining MIC values, $10 \mu \mathrm{l}$ of the contents was aspirated from the wells which showed no growth according to MIC results and inoculated on Mueller Hinton Agar. The plates were incubated at $37{ }^{\circ} \mathrm{C}$ for 24 hours. The plates were examined for the presence/absence of the growth of the bacteria.
The MBC values were determined as the lowest concentration of the antimicrobial agent which inhibits the growth of bacteria.

\section{Results}

In our study, the effects of hormones on MIC values of ciprofloxacin and gentamicin for MRSA and $P$. aeruginosa were determined separately and together.

MIC and MBC values of ciprofloxacin and gentamicin for MRSA were 320 and 160 $\mu \mathrm{g} / \mathrm{ml}$, respectively without hormones. As shown in Table 1, hormones decreased the MIC values of both ciprofloxacin and gentamicin for MRSA strain. The alterations of MIC values were almost 2-64 fold. Although $\mathrm{MBC}$ values of gentamicin were found to be reduced in the presence of hormones, ciprofloxacin did not change except for high concentrations of melatonin.

Table 1. MIC and MBC values of ciprofloxacin and gentamicin $(\mu \mathrm{g} / \mathrm{ml})$ for MRSA with and without hormones

\begin{tabular}{lccccc}
\hline Antibiotics & $\begin{array}{c}\text { without } \\
\text { hormones }\end{array}$ & HNE & HMEL & LNE & LMEL \\
\hline Ciprofloxacin & 320 & 160 & 80 & 160 & 80 \\
Gentamicin & 160 & $\leq 2.5$ & $\leq 2.5$ & $\leq 2.5$ & $\leq 2.5$ \\
Ciprofloxacin & 320 & 320 & 160 & 320 & 320 \\
Gentamicin & 160 & $\leq 2.5$ & 20 & 10 & $\leq 2.5$ \\
\hline
\end{tabular}

HNE: high norepinephrine, LNE: low norepinephrine, HMEL: high melatonin, LMEL: low melatonin

Table 2. MIC and MBC values of ciprofloxacin and gentamicin $(\mu \mathrm{g} / \mathrm{ml})$ for P. aeruginosa with and without hormones

\begin{tabular}{lcllcc}
\hline Antibiotics & $\begin{array}{c}\text { without } \\
\text { hormones }\end{array}$ & HNE & HMEL & LNE & LMEL \\
\hline Ciprofloxacin & 2.5 & 2.5 & 2.5 & 2.5 & 2.5 \\
Gentamicin & 2.5 & 2.5 & 2.5 & 2.5 & 2.5 \\
Ciprofloxacin & 2.5 & 2.5 & 2.5 & 5 & 2.5 \\
Gentamicin & 2.5 & 2.5 & 2.5 & 5 & 2.5 \\
\hline
\end{tabular}

HNE: high norepinephrine, LNE: low norepinephrine, HMEL: high melatonin, LMEL: low melatonin 
Table 3. MIC and MBC values of ciprofloxacin and gentamicin $(\mu \mathrm{g} / \mathrm{ml})$ for MRSA+ P. aeruginosa in co-culture conditions with and without hormones

\begin{tabular}{lccccc}
\hline Antibiotics & $\begin{array}{c}\text { without } \\
\text { hormones }\end{array}$ & HNE & HMEL & LNE & LMEL \\
\hline Ciprofloxacin & 20 & 10 & MIC values \\
Gentamicin & 40 & 2.5 & 2.5 & 5 & 2.5 \\
Ciprofloxacin & 40 & 160 & 2.5 & 2.5 & 2.5 \\
Gentamicin & 40 & 2.5 & 2.5 & 40 & 40 \\
\hline
\end{tabular}

HNE: high norepinephrine, LNE: low norepinephrine, HMEL: high melatonin, LMEL: low melatonin

MIC and MBC values of ciprofloxacin and gentamicin for $P$. aeruginosa were determined as $2.5 \mu \mathrm{g} / \mathrm{ml}$ without hormones. No alterations were found in MIC and MBC values of ciprofloxacin and gentamicin. On the other hand, the presence of norepinephrine at low concentration was found to be 2-fold increased the MBC values of both antibiotics (Table 2).

In our study, we also examined MIC and MBC values of antibiotics in co-culture conditions with and without hormones. In the absence of hormones, MIC values of ciprofloxacin and gentamicin were determined as 20 and $40 \mu \mathrm{g} / \mathrm{ml}$, respectively; MBC values of both antibiotics were determined as 40 $\mu \mathrm{g} / \mathrm{ml}$. Furthermore, it was found that hormones decreased (2-16 fold) MIC values of both antibiotics and MBC value of gentamicin was found to be reduced (8-16 fold) in the presence of hormones, in co-culture conditions. Whereas high melatonin decreased MIC value of ciprofloxacin, high norepinephrine increased it. There was no alteration of MIC values of ciprofloxacin in the presence of low hormone concentrations (Table 3).

As clearly seen in Table $\mathbf{1}$ and Table 2, MIC and MBC values of antibiotics for monoculture were found to be changed compared to co-culture conditions. MIC values of ciprofloxacin and gentamicin (320 and 160 $\mu \mathrm{g} / \mathrm{ml}$, respectively) for $S$. aureus-monoculture were found to be reduced (ciprofloxacin and gentamicin were 20 and $40 \mu \mathrm{g} / \mathrm{ml}$ ) compared to co-culture conditions. Similarly, MBC values of both antibiotics (ciprofloxacin and gentamicin were 320 and $160 \mu \mathrm{g} / \mathrm{ml}$ ) decreased for $S$. aureus strain compared to co-cultures' MBC values (ciprofloxacin and gentamicin were $40 \mu \mathrm{g} / \mathrm{ml}$ ) (Table 1). On the other hand, it was found that MIC and MBC values of ciprofloxacin and gentamicin $(2.5 \mu \mathrm{g} / \mathrm{ml})$ for monocultures of $P$. aeruginosa were found to be increased compared to co-culture conditions (Table 2).

\section{Discussions}

Lyte and Ernst introduced the concept of microbial endocrinology, a bi-directional interaction between host and microbe through interkingdom signaling based on neuroendocrine factors of human and sensor molecules of microorganisms which have coexisted and known each other for millions of years (Lyte M and Ernst S., 1992; Sharaff and Freestone, 2011). Since then, many studies have shown the effects of host hormones, as environmental factors in the host body, on bacterial physiology during the infectious processes (Kornman and Loesche, 1982; Plotkin and Viselli, 2000; Lyte Mark, 2010; Alves et al., 2014; Gumus et al., 2017; Gümüş et al., 2019; Truccollo et al., 2020; Vidaillac et al., 2020; Engelsöy et al., 2021).

Beyond many physiological features of bacteria, antibiotic susceptibility can also be modulated by hormones helping bacteria for 
better growth recovery following antibiotic treatment. Freestone et al., 2012 have indicated that $P$. aeruginosa exposed to sub-inhibitory concentrations of tobramycin in serum-SAPI medium still growth when NE was added. This effect has also been observed in S. epidermidis (Freestone et al., 2016). Ambrose et al., 2018 concluded that $\mathrm{NE}$ in combination with levofloxacin increased the rate of Escherichia coli replication provides an opportunity to enhance the bactericidal effect. On the contrary, it was reported that NE markedly decreased antibacterial activity of tigecycline against multidrug-resistant Acinetobacter baumanii strain, but had only a slight effect on the activity of colistin (Inaba et al., 2016).

A mammalian hormone, melatonin (MEL) plays roles as antioxidant, anti-inflammatory, and immunomodulatory; there is a limited number of studies about the possible effects of MEL on microbial behaviors (Tekbas et al., 2008; Bishayi et al., 2016; Zhou et al., 2016; Lee et al., 2018, 2020; Chen et al., 2019; Ganganna et al., 2021). Wiid et al., 1999 suggested that for the treatment of tuberculosis, dosing isoniazid simultaneous with MEL provides at least a threefold increase in the efficacy of the drug in-vitro. Kilinçel et al., 2019 observed similar results for antifungals against Candida species; they have shown that MEL in combination with antifungals reduces the MIC values of antifungals. Liu et al., 2020 determined that MEL exhibits synergistic activity with colistin against resistant pathogens both in-vitro and in-vivo.

Consistent with earlier studies as mentioned above, in our study, we found that $\mathrm{NE}$ and MEL, each at two different concentrations, decreased MIC values of ciprofloxacin and gentamicin, and decreased MBC value of gentamicin for MRSA strain. However, MBC value of ciprofloxacin was found to be reduced in the presence of only MEL at high concentration.
For $P$. aeruginosa, there wasn't any difference in antibiotic's MICs. For MBC of both antibiotics, only an increasing was found to be detected in the presence of NE at low concentration. Considering these results, indicating possible effects of hormones on antimicrobial susceptibility depend on hormones' concentrations, antibiotics and bacterial species tested.

MRSA and $P$. aeruginosa are commonly identified as causative agents of polymicrobial infections. Antibacterial treatment of polymicrobial infections can be a challenge, thus there are many studies investigating microbial behaviors and interactions during the polymicrobial infectious processes of $S$. aureus and $P$. aeruginosa (Beaudoin et al., 2017; Orazi and O'Toole, 2017; Radlinski et al., 2017; Tognon et al., 2017; Alves et al., 2018; Kahl, 2018; Briaud et al., 2019, 2020a; Orazi G, Jean-Pierre F, 2020; Yung et al., 2021; Camus et al., 2021).

Although the pathogens are studied in pure culture, it is well known that microorganisms within diverse communities are actively responding to each other. These interactions between species can affect pathogenic behaviors such as virulence, biofilm formation and antibiotic tolerance (Korgaonkar et al., 2013; Vega et al., 2013; Briaud et al., 2019, 2020b). Therefore, our study was carried out to assess antibiotic susceptibilities of MRSA and $P$. aeruginosa against ciprofloxacin and gentamicin also in co-culture conditions. Whereas, MIC and MBC values of both antibiotics for co-culture were found to be reduced compared to monoculture of MRSA; were found to be increased compared to monoculture of $P$. aeruginosa.

There are many studies examining possible mechanisms of alterations in antibiotic susceptibilities in microbial co-existence (Michelsen et al., 2014; Beaudoin et al., 2017; Radlinski et al., 2017; Briaud et al., 2019; 
Trizna et al., 2020; Dehbashi et al., 2021). In such a study, it was shown that antibiotic resistance increased via composing polymicrobial biofilm (Beaudoin et al., 2017). In another study, it was found that $S$. aureus co-cultured with $P$. aeruginosa in a wound-like medium, had higher tolerance to tetracycline and gentamicin compared to its single species culture but tolerance to ciprofloxacin was reported to be not changed. Besides, the antibiotic tolerance of $P$. aeruginosa was not changed (DeLeon et al., 2014). Although has not been clarified the specific mechanism, some authors suggested that excreted enzymes of $P$. aeruginosa could produce several aminoglycoside-modifying enzymes (Poole, 2005; DeLeon et al., 2014), which could have inactivated the gentamicin in the co-culture which protects both species.

All these kinds of studies suggest that today, to manage antibiotic resistance, a global health concern, inter-kingdom interactions can be used as a target for new antibacterial therapeutics.

It is still unclear whether NE and MEL have some effects on antibiotic susceptibilities of co-cultured microorganisms. To our knowledge, this is the first report aimed to investigate the effects of hormones on microbial behaviors in co-culture conditions. We found that hormones decreased MIC values when bacteria were grown together. While MBC values of both antibiotics were reduced in the presence of MEL at high concentrations, MBC value of ciprofloxacin was increased in the presence of a high level of NE. On the other hand, low levels of hormones did not affect MBC values of ciprofloxacin. All hormones also decreased MBC values of gentamicin.

\section{Conclusions}

In conclusion, our study clearly indicated that, NE and MEL affect antibiotic susceptibilities of MRSA and $P$. aeruginosa strains in mono and co-culture. These possible effects of hormones on bacterial susceptibilities are needed to be investigated furtherly, especially which could be useful for developing new approaches for the treatment of infectious diseases.

\section{Conflict of interest}

The authors declare that there are no conflicts of interest related to this article.

\section{References}

1. Alves CT, Silva S, Pereira L, Williams DW, Azeredo J, Henriques M (2014) Effect of progesterone on Candida albicans vaginal pathogenicity. Inter $\mathbf{J}$ Med Microbiol 304:1011-1017.

2. Alves PM, Al-Badi E, Withycombe C, Jones PM, Purdy KJ, Maddocks SE (2018) Interaction between Staphylococcus aureus and Pseudomonas aeruginosa is beneficial for colonisation and pathogenicity in a mixed biofilm. Pathog Dis 76:1-10.

3. Ambrose PG, VanScoy BD, Adams J, Fikes S, Bader JC, Bhavnani SM, Rubino CM (2018) Norepinephrine in combination with antibiotic therapy increases both the bacterial replication rate and bactericidal activity. Antimicrob Agents Chemother 62(4): e02257-17.

4. Bansal T, Englert D, Lee J, Hegde M, Wood TK, Jayaraman A (2007) Differential effects of epinephrine, norepinephrine, and indole on Escherichia coli O157:H7 chemotaxis, colonization, and gene expression. Infect Immun 75:4597-4607.

5. Bearson BL (2016) Molecular profiling: Catecholamine modulation of gene 
expression in escherichia coli o157:H7 and salmonella enterica serovar typhimurium. Adv Experiment Med Biol 874:167-182.

6. Beaudoin T, Yau YCW, Stapleton PJ, Gong Y, Wang PW, Guttman DS, Waters V (2017) Staphylococcus aureus interaction with Pseudomonas aeruginosa biofilm enhances tobramycin resistance. npj Biofilms Microbiomes 3:1-8.

7. Belay T, Aviles H, Vance M, Fountain K, Sonnenfeld G (2003) Catecholamines and in vitro growth of pathogenic bacteria: Enhancement of growth varies greatly among bacterial species. Life Sci 73:15271535.

8. Bishayi B, Adhikary R, Nandi A, Sultana S (2016) Beneficial Effects of Exogenous Melatonin in Acute Staphylococcus aureus and Escherichia coli Infection-Induced Inflammation and Associated Behavioral Response in Mice After Exposure to Short Photoperiod. Inflammation 39:2072-2093.

9. Boukerb AM, Cambronel M, Rodrigues S, Mesguida O, Knowlton R, Feuilloley MGJ, Zommiti M, Connil N (2021) InterKingdom Signaling of Stress Hormones: Sensing, Transport and Modulation of Bacterial Physiology. Frontiers in Microbiology 12:1-18.

10. Boyanova L (2017) Stress hormone epinephrine (adrenaline) and norepinephrine (noradrenaline) effects on the anaerobic bacteria. Anaerobe 44:13-19.

11. Briaud P, Bastien S, Camus L, Boyadjian M, Reix P, Mainguy C, Vandenesch F, Doléans-Jordheim A, Moreau K (2020a) P131 Most of Staphylococcus aureus and Pseudomonas aeruginosa coinfecting isolates coexist, a condition that may impact clinical outcomes in cystic fibrosis patients. Front Cell Infect Microbiol 3:10:266. doi: 10.3389/fcimb.2020.00266.

12. Briaud P, Bastien S, Camus L, Boyadjian M, Reix P, Mainguy C, Vandenesch F,
Doléans-Jordheim A, Moreau K (2020b) Impact of Coexistence Phenotype Between Staphylococcus aureus and Pseudomonas aeruginosa Isolates on Clinical Outcomes Among Cystic Fibrosis Patients. Front in Cell Infect Microbiol 10:1-10.

13. Briaud P, Camus L, Bastien S, DoléansJordheim A, Vandenesch F, Moreau K (2019) Coexistence with Pseudomonas aeruginosa alters Staphylococcus aureus transcriptome, antibiotic resistance and internalization into epithelial cells. Sci Rep 9:1-14.

14. Camus L, Briaud P, Vandenesch F, Moreau K (2021) How Bacterial Adaptation to Cystic Fibrosis Environment Shapes Interactions Between Pseudomonas aeruginosa and Staphylococcus aureus. Front Microbiol 12:1-16.

15. Chen X, Sun C, Laborda P, He Y, Zhao Y, Li C, Liu F (2019) Melatonin treatments reduce the pathogenicity and inhibit the growth of Xanthomonas oryzae pv. oryzicola. Plant Pathol 68:288-296.

16. CLSI, Clinical and Laboratory Standards Institute, M07- A10: Methods for Dilution Antimicrobial Susceptibility Tests for Bacteria That Grow Aerobically; Approved Standard, Vol. 35, CLSI, Wayne, PA, USA, $31^{\text {th }}$ edition, 2021

17. Dalton T, Dowd SE, Wolcott RD, Sun Y, Watters C, Griswold JA, Rumbaugh KP (2011) An in vivo polymicrobial biofilm wound infection model to study interspecies interactions. PLoS ONE 2011;6(11):e27317.

doi: 10.1371/journal.pone.0027317.

18. Dehbashi S, Alikhani MY, Tahmasebi H, Arabestani MR (2021) The inhibitory effects of Staphylococcus aureus on the antibiotic susceptibility and virulence factors of Pseudomonas aeruginosa: A549 cell line model. AMB Express 30;11(1):50. doi: 10.1186/s 13568-021-01210-y. 
19. DeLeon S, Clinton A, Fowler H, Everett J, Horswill AR, Rumbaugh KP (2014) Synergistic interactions of Pseudomonas aeruginosa and Staphylococcus aureus in an In vitro wound model. Infect Immun 82:4718-4728.

20. Engelsöy U, Svensson MA, Demirel I (2021) Estradiol Alters the Virulence Traits of Uropathogenic Escherichia coli. Front Microbiol 20;12:682626.

doi: 10.3389/fmicb.2021.682626.

21. Freestone PP, Hirst RA, Sandrini SM, Sharaff F, Fry H, Hyman S, O'Callaghan C (2012) Pseudomonas aeruginosacatecholamine inotrope interactions: A contributory factor in the development of ventilator-associated pneumonia? Chest 142:1200-1210.

22. Freestone PPE, Al-Dayan N, Lyte M (2016) Staphylococci, catecholamine inotropes and hospital-acquired infections. Adv Exp Med Biol 874:183-199.

23. Freestone PPE, Haigh RD, Lyte M (2007) Specificity of catecholamine-induced growth in Escherichia coli O157:H7, Salmonella enterica and Yersinia enterocolitica. FEMS Microbiol Lett 269:221-228.

24. Fteita D, Könönen E, Söderling E, Gürsoy UK (2014) Effect of estradiol on planktonic growth, coaggregation, and biofilm formation of the Prevotella intermedia group bacteria. Anaerobe 27:7-13.

25. Ganganna A, Rudariah CB, Rao R, Prakash VM (2021) Antibacterial activity of melatonin against prime periodontal pathogens: An in vitro study. J Intern Oral Health 13:164-168.

26. Gonçalves B, Bernardo R, Wang C, Schröder MS, Pedro NA, Butler G, Azeredo J, Henriques M, Pereira Mira N, Silva S (2020) Effect of progesterone on Candida albicans biofilm formation under acidic conditions: A transcriptomic analysis. Intern J Med Microbiol 310:151414.

27. Gümüş D, Kalaycı Yüksek F, Sefer Ö, Yörük E, Uz G, Anğ Küçüker M (2019) The roles of hormones in the modulation of growth and virulence genes' expressions in UPEC strains. Microb Pathog 132:319324.

28. Gumus D, Yoruk E, Kalayci-Yuksek F, Uz G, Topal-Sarikaya A, Ang-Kucuker M (2017) The effects of insulin and glucose on different characteristics of a UPEC: Alterations in growth rate and expression levels of some virulence genes. Clin Lab 63:1589-1597.

29. He F, Wu X, Zhang Q, Li Y, Ye Y, Li P, Chen S, Peng Y, Hardeland R, Xia Y (2021) Bacteriostatic Potential of Melatonin: Therapeutic Standing and Mechanistic Insights. Front Immunol 12.

30. Inaba M, Matsuda N, Banno H, Jin W, Wachino J ichi, Yamada K, Kimura K, Arakawa Y (2016) In vitro reduction of antibacterial activity of tigecycline against multidrug-resistant

Acinetobacter baumannii with host stress hormone norepinephrine. Intern J Antimicrob Agents 48:680-689.

31. Kahl BC (2018) Staphylococcus aureus and Pseudomonas aeruginosa Respiratory Tract Coinfection - What Can We Learn from Animal Models? J Infect Dis 217:854-856.

32. Kilinçel Ö, Çalişkan E, Şahin I, Öztürk CE, Kiliç N, Öksüz Ş (2019) The effect of melatonin on antifungal susceptibility in planktonic and biofilm forms of Candida strains isolated from clinical samples. Med Mycol 57:45-51.

33. Kim S, Yoon Y, Choi KH (2015) Pseudomonas aeruginosa DesB promotes staphylococcus aureus growth inhibition in coculture by controlling the synthesis of HAQs. PLoS ONE 10:1-16. 
34. Korgaonkar A, Trivedi U, Rumbaugh KP, Whiteley M (2013) Community surveillance enhances Pseudomonas aeruginosa virulence during polymicrobial infection. Proc Natl Acad Sci U S A 110:1059-1064.

35. Kornman KS, Loesche WJ (1982) Effects of estradiol and progesterone on Bacteroides melaninogenicus and Bacteroides gingivalis. Infect Immun 35:256-263.

36. Lee SJ, Lee HJ, Jung YH, Kim JS, Choi SH, Han HJ (2018) Melatonin inhibits apoptotic cell death induced by Vibrio vulnificus VvhA via melatonin receptor 2 coupling with NCF-1 article. Cell Death Dis 19;9(2):48. doi: 10.1038/s41419-0170083-7.

37. Lee YM, Park JP, Jung YH, Lee HJ, Kim JS, Choi GE, Han HJ, Lee SJ (2020) Melatonin restores Muc2 depletion induced by V. vulnificus VvpM via melatonin receptor 2 coupling with Gaq. J Biomed Sci 27:1-14.

38. Li W, Lyte M, Freestone PP, Ajmal A, Colmer-Hamood J HA (2009) Norepinephrine represses the expression of toxA and the siderophore genes in Pseudomonas aeruginosa. FEMS Microbiol Lett 299(1):100-9. doi: 10.1111/j.15746968.2009.01739.x

39. Liu Y, Jia Y, Yang K, Tong Z, Shi J, Li R, Xiao X, Ren W, Hardeland R, Reiter RJ, Wang Z, Wang Z, Ren W, Liu Y (2020) Melatonin overcomes MCR-mediated colistin resistance in Gram-negative pathogens. Theranostics 10:10697-10711.

40. Lucca V, Apellanis Borges K, Quedi Furian T, Borsoi A, Pippi Salle CT, de Souza Moraes HL, Pinheiro do Nascimento V (2020) Influence of the norepinephrine and medium acidification in the growth and adhesion of Salmonella Heidelberg isolated from poultry. Microb Pathog 138:103799.
41. Lyte JM, Shrestha S, Wagle BR, Liyanage R, Martinez DA, Donoghue AM, Daniels KM, Lyte M (2021) Serotonin modulates Campylobacter jejuni physiology and in vitro interaction with the gut epithelium. Poultry Sci 100(3):100944. doi: 10.1016/j.psj.2020.12.041.

42. Lyte M and Ernst S. (1992) Catecholamine induced growth of gram negative bacteria. Life Sci 50:203-212.

43. Lyte Mark FPPE (2010) Microbial Endocrinology. Springer, New York

44. Lyte, Mark, Cryan JF (eds) (2014) Microbial Endocrinology: The MicrobiotaGut-Brain Axis in Health and Disease, Springer, New York

45. Michelsen CF, Christensen AMJ, Bojer MS, Høiby N, Ingmer H, Jelsbak L (2014) Staphylococcus aureus alters growth activity, autolysis, and antibiotic tolerance in a human host-adapted Pseudomonas aeruginosa lineage. J Bacteriol 196:39033911.

46. Murray JL, Connell JL, Stacy A, Turner KH, Whiteley M (2014) Mechanisms of synergy in polymicrobial infections. J Microbiol 52:188-199.

47. Nguyen AT, Oglesby-Sherrouse AG (2016) Interactions between Pseudomonas aeruginosa and Staphylococcus aureus during co-cultivations and polymicrobial infections. Appl Microbiol Biotechnol 100:6141-6148.

48. Orazi G, Jean-Pierre F OG (2020) Pseudomonas aeruginosa PA14 Enhances the Efficacy of Norfloxacin against Staphylococcus aureus Newman Biofilms Giulia. J Bacteriol 25;202(18):e0015920. doi: 10.1128/JB.00159-20.

49. Orazi G, O’Toole GA (2017) Pseudomonas aeruginosa alters Staphylococcus aureus sensitivity to vancomycin in a biofilm model of cystic fibrosis infection. mBio $8: 1-17$. 
50. Ping O, Mao S, Xuewen H, Kaiyu W, Zhongqiong Y, Hualin F, Yinglun L, Yi G, Gang S, Changliang $H$, Xiaoxia L, Weiming L, Lixia L, Yunfeng Z, Xu S, Lizi Y (2017) Sclareol protects Staphylococcus aureus-induced lung cell injury via inhibiting alpha-hemolysin expression. J Microbiol Biotechnol 27:19-25.

51. Plotkin BJ, Roose RJ, Erikson Q, Viselli SM (2003) Effect of Androgens and Glucocorticoids on Microbial Growth and Antimicrobial Susceptibility. Curr Microbiol 47:514-520.

52. Plotkin BJ, Viselli SM (2000) Effect of insulin on microbial growth. Curr Microbiol 41:60-64.

53. Poole K (2005) Aminoglycoside resistance in Pseudomonas aeruginosa. Antimicrob Agents Chemother 49:479-487.

54. Radlinski L, Rowe SE, Kartchner LB, Maile R, Cairns BA, Vitko NP, Gode CJ, Lachiewicz AM, Wolfgang MC, Conlon BP (2017) Pseudomonas aeruginosa exoproducts determine antibiotic efficacy against Staphylococcus aureus. PLoS Biology 15:1-25.

55. Sandrini S, Alghofaili F, Freestone P, Yesilkaya H (2014) Host stress hormone norepinephrine stimulates pneumococcal growth, biofilm formation and virulence gene expression. BMC Microbiol 14:1-12.

56. Sharaff F, Freestone P (2011) Microbial Endocrinology. Cent Euro J Biol 6:685694.

57. Tekbas OF, Ogur R, Korkmaz A, Kilic A, Reiter RJ (2008) Melatonin as an antibiotic: New insights into the actions of this ubiquitous molecule. J Pineal Res 44:222226.

58. Tognon M, Köhler T, Gdaniec BG, Hao Y, Lam JS, Beaume M, Luscher A, Buckling A, Van Delden C (2017) Co-evolution with Staphylococcus aureus leads to lipopolysaccharide alterations in
Pseudomonas aeruginosa. ISME J 11:22332243.

59. Trizna EY, Yarullina MN, Baidamshina DR, Mironova A V., Akhatova FS, Rozhina E V., Fakhrullin RF, Khabibrakhmanova AM, Kurbangalieva AR, Bogachev MI, Kayumov AR (2020) Bidirectional alterations in antibiotics susceptibility in Staphylococcus aureus-Pseudomonas aeruginosa dual-species biofilm. Sci Rep 10:1-18.

60. Truccollo B, Whyte P, Bolton DJ (2020) An investigation of the effect of catecholamines and glucocorticoids on the growth and pathogenicity of Campylobacter jejuni. Pathogens 9:1-15.

61. Vega NM, Allison KR, Samuels AN, Klempner MS, Collins JJ (2013) Salmonella typhimurium intercepts Escherichia coli signaling to enhance antibiotic tolerance. Proc Natl Acad Sci U S A. 110:14420-14425.

62. Vidaillac C, Yong VFL, Aschtgen MS, Qu J, Yang S, Xu G, Seng ZJ, Brown AC, Ali MK, Jaggi TK, Sankaran J, Foo YH, Righetti F, Nedumaran AM, Aogáin M Mac, Roizman D, Richard JA, Rogers TR, Toyofuku M, Luo D, Loh E, Wohland T, Czarny B, Horvat JC, Hansbro PM, Yang $\mathrm{L}$, Li L, Normark S, Normark BH, Chotirmall SH (2020) Sex steroids induce membrane stress responses and virulence properties in Pseudomonas aeruginosa. mBio 11:1-19.

63. Wiid I, Hoal-Van Helden E, Hon D, Lombard C, Van Helden P (1999) Potentiation of isoniazid activity against Mycobacterium tuberculosis by melatonin. Antimicrob Agents Chemother 43:975977.

64. Wijesinghe G, Dilhari A, Gayani B, Kottegoda N, Samaranayake L, Weerasekera M (2019) Influence of Laboratory Culture Media on in vitro 
Growth, Adhesion, and Biofilm Formation of Pseudomonas aeruginosa and Staphylococcus aureus. Med Prin Pract 28:28-35.

65. Xu F, Wu C, Guo F, Cui G, Zeng X, Yang B, Lin J (2015) Transcriptomic analysis of Campylobacter jejuni NCTC 11168 in response to epinephrine and norepinephrine. Front Microbiol 6:1-11.

66. Yang L, Liu Y, Markussen T, Høiby N, Tolker-Nielsen T, Molin S (2011) Pattern differentiation in co-culture biofilms formed by Staphylococcus aureus and Pseudomonas aeruginosa. FEMS Immunol Med Microbiol 62:339-347.

67. Yang Q, Anh NDQ, Bossier P, Defoirdt T (2014) Norepinephrine and dopamine increase motility, biofilm formation, and virulence of Vibrio harveyi. Front Microbiol 5:1-12.

68. Yung DBY, Sircombe KJ, Pletzer D (2021) Friends or enemies? The complicated relationship between Pseudomonas aeruginosa and Staphylococcus aureus. Mol Microbiol 116:1-15.

69. Zhou W, Zhang X, Zhu CL, He ZY, Liang JP, Song ZC (2016) Melatonin receptor agonists as the "perioceutics" agents for periodontal disease through modulation of Porphyromonas gingivalis virulence and inflammatory response. PLoS ONE 10;11(11):e0166442.

doi: 10.1371/journal.pone.0166442. 